\title{
Lebensqualität und Dorfentwicklung. Eine Fallstudie aus Niedersachsen
}

\author{
Ulrich Harteisen $^{1} \cdot$ Swantje Eigner-Thiel $^{1}$
}

Eingegangen: 29. Juli 2016 / Angenommen: 26. Oktober 2016 / Online publiziert: 23. November 2016

(c) Springer-Verlag Berlin Heidelberg 2016

Zusammenfassung Vor dem Hintergrund des demographischen Wandels wird aktuell intensiv darüber diskutiert, wie die Lebensqualität in schrumpfenden Dörfern erhalten werden kann. In diesem Beitrag wird eine raumwissenschaftliche Perspektive eingenommen und mit dem psychologischen Konzept der Lebensqualität verknüpft. Die Ergebnisse einer qualitativen empirischen Untersuchung, in der 43 Dorfbewohner in vier niedersächsischen Dörfern danach gefragt wurden, wie sie selbst ihre Lebensqualität im Dorf bewerten und wo sie Handlungsbedarf, eigene Gestaltungsmöglichkeiten und Unterstützungsbedarf sehen, werden in Bezug gesetzt zu bestehenden und neuen Ansätzen der Dorfentwicklung. Im Ergebnis wird deutlich, dass die Dorfbewohner den sozialen Kontakten im Dorf eine hohe Bedeutung beimessen und dass diese einerseits direkt zur eigenen Lebenszufriedenheit beitragen, andererseits aber auch eine Basis für die gemeinschaftliche Gestaltung und Schaffung einer guten Lebensqualität im Dorf darstellen. Ausgehend von diesen Ergebnissen wird empfohlen, soziale Fragen der Dorfentwicklung in der Dorferneuerungsplanung stärker zu verankern sowie neue unterstützende Angebote weiter zu entwickeln. Insgesamt unterstreichen die Ergebnisse der Forschung die Bedeutung der zivilgesellschaftlichen Kraft der Dorfgemeinschaft, die als ein bedeu-

Prof. Dr. Ulrich Harteisen

ulrich.harteisen@hawk-hhg.de

Dr. Swantje Eigner-Thiel

swantje.eigner-thiel@hawk-hhg.de

1 Fakultät Ressourcenmanagement, HAWK - Hochschule für angewandte Wissenschaft und Kunst Hildesheim, Holzminden, Göttingen, Büsgenweg 1a, 37077 Göttingen, Deutschland tendes endogenes Potenzial eine stärkere Beachtung in der Dorfentwicklung verdient.

Schlüsselwörter Dorfforschung · Lebensqualität · Dorfentwicklung · Demographischer Wandel · Ländliche Entwicklung

\section{Quality of Life and Village Development: A Case Study from Lower Saxony, Germany}

Abstract In the context of demographic change there is currently an intensive discussion about how the quality of life can be maintained in villages with a shrinking population. In this paper, a spatial perspective is linked to the psychological concept of quality of life. The results of the qualitative empirical study in which 43 villagers in four Lower Saxony villages were asked how they themselves assess their quality of life in the village and where they see a need for action, their own opportunities for creativity and need for support are juxtaposed with existing and new approaches to village development. The result clearly shows that the village inhabitants attach great importance to social contacts in the village and that these, on the one hand, contribute directly to their own satisfaction with life, and on the other, also importantly constitute a basis for jointly shaping community matters and establishing good quality of life in the village. Based on these results, the authors recommend integrating social questions of village development more emphatically into village renewal plans, as well as further developing new support services. Overall, the research results emphasize the importance of the village community as a stimulus in civil society that deserves more attention as an important endogenous potential within village development. 
Keywords Research into village life - Quality of life · Village development $\cdot$ Demographic change $\cdot$ Rural development

\section{Einleitung}

In vielen Dörfern stellt sich vor dem Hintergrund des demographischen und wirtschaftsstrukturellen Wandels die Frage, ob und wie die technische und soziale Infrastruktur zur Sicherung der Lebensqualität zukünftig gewährleistet werden kann. Nahversorgung, Mobilität, Bildung und Gesundheitsversorgung sind die Themenfelder, die in der Forschung zu ländlichen Räumen aktuell intensiv diskutiert werden (vgl. Wilde 2014; Küpper/Scheibe 2015; Steinführer 2015).

Diese Entwicklungen bewegen auch die Menschen in den Dörfern. Was liegt näher, als in den Dörfern die Dorfbewohner selbst nach der Bewertung ihrer Lebensqualität zu befragen, so geschehen im Teilprojekt „Lebensqualität im Dorf" im Forschungsschwerpunkt DIALOG (gefördert von Januar 2012 bis Dezember 2016 aus Landesmitteln des Niedersächsischen $\operatorname{Vorab}^{1}$ ), in dem es darum geht, die Folgen des demographischen Wandels und die Umgangsweise damit aus der Sicht verschiedener Disziplinen zu analysieren und Ansätze zur Gestaltung der Veränderungsprozesse zu entwickeln. Abbildung 1 stellt die Vorgehensweise des Forschungsvorhabens dar.

Ausgehend von den Ergebnissen der qualitativen Forschung aus diesem Forschungsprojekt wird die Frage diskutiert, wie Lebensqualität in Dörfern zukünftig bewahrt werden kann und welche Rolle dabei die Dorferneuerung sowie neue Instrumente spielen sollten. Die Diskussion knüpft an die Forschung zur Weiterentwicklung der Dorferneuerung (Ritzinger 2011: 88) und zur Bedeutung von „governance-strukturierten Problemlösungsansätzen“ im Dorf (Born 2009: 61) an und geht der Frage nach, ob die bestehenden Instrumente und Methoden der Dorfentwicklung ausreichen, um die Lebensqualität in den Dörfern nachhaltig zu sichern (vgl. auch Kapitel 2.2).

Das Dorf als Lebensraum steht im Mittelpunkt dieses Beitrags, daher ist es einleitend wichtig, den Begriff zu definieren. Was ist ein Dorf? Da es auf diese Frage keine einfache Antwort gibt, werden hier einige Merkmale zusammengestellt:

- Kriterium Siedlungsgröße: Henkel (1995: 31) beschreibt das Dorf als eine relativ kleine ländliche Gruppensiedlung. Relativ klein bedeutet aber auch, dass eine scharfe
Abgrenzung über das Kriterium „Größe“ nicht möglich ist. So gibt es kleine Landstädte und große Dörfer, die ähnliche Einwohnerzahlen aufweisen.

- Kriterium Baukultur: Dörfer unterscheiden sich von Städten mehr oder weniger durch ihre Baukultur. Auch wenn Dörfer heute kaum noch durch die Landwirtschaft als Erwerbszweig geprägt sind, so ist doch in vielen Dörfern die Bausubstanz, die ursprünglich der Landwirtschaft und dem ländlichen Handwerk diente, noch ortsbildprägend (Henkel 1995: 29). Allerdings ist auch die historische Baukultur nicht mehr in allen Dörfern sichtbar, da sie oft im Zuge von Dorfmodernisierungen verschwunden ist.

- Kriterium Dorfgemeinschaft: Die lokale Gemeinschaft mit ausgeprägten Nachbarschafts- und Verwandtschaftsbeziehungen ist ein weiteres wichtiges Merkmal von Dörfern (Krambach 2013: 9) und vielfach bis heute lebendig.

Die drei hier aufgeführten dorfprägenden Merkmale charakterisieren in der Zusammenschau den Siedlungstyp Dorf, unterliegen aber auch stetigen Wandlungsprozessen. Ein Dorf kann somit zusammenfassend als eine ,historisch gewachsene und ursprünglich meist agrarisch geprägte, relativ kleine und überschaubare Siedlung mit eigenem Ortsnamen" beschrieben werden (Krambach 2013: 9 f.). Von besonderer Bedeutung ist die Dorfgemeinschaft, die auf einer „spezifischen Intensität der sozialen Kontakte“ und einer historisch gewachsenen „Gemeinsamkeit der lokalen Existenzbedingungen“ beruht, die auch heute noch Grundlage für ein gemeinsames Interesse an einer Bewahrung und Schaffung einer guten Lebensqualität sein können (Krambach 2013: 10).

Aber was ist wichtig und was eher unbedeutend für eine gute Lebensqualität? Zu diesem Thema sollen die Dorfbewohner als ,Dorfexperten“ kompetent Auskunft geben. Ziel dieses Beitrags ist es daher, aus akteurzentrierter Sicht Aussagen und Einschätzungen zur Lebensqualität in den Dörfern darzustellen und diese Erkenntnisse in die Diskussion um die Weiterentwicklung der Instrumente und Methoden der Dorfentwicklung einzuspeisen. Damit stehen zwei Forschungsfragen im Fokus:

- Wie stellt sich die Lebensqualität auf dem Land, speziell in Dörfern, aus der Sicht ihrer Bewohner dar?

- Welche Schlussfolgerungen können aus den Aussagen der Dorfbewohner für die Diskussion um die Weiterentwicklung der Instrumente und Methoden der Dorfentwicklung abgeleitet werden?

${ }^{1} \mathrm{Vgl}$. http://www.mwk.niedersachsen.de/startseite/forschung/ forschungsfoerderung/forschungsfoerderung-durch-das-mwk118860.html (24.10.2016). 
Abb. 1: Vorgehensweise im Forschungsvorhaben „Lebensqualität im Dorf“
Forschungsgegenstand

Lebensqualität und Dorfentwicklung

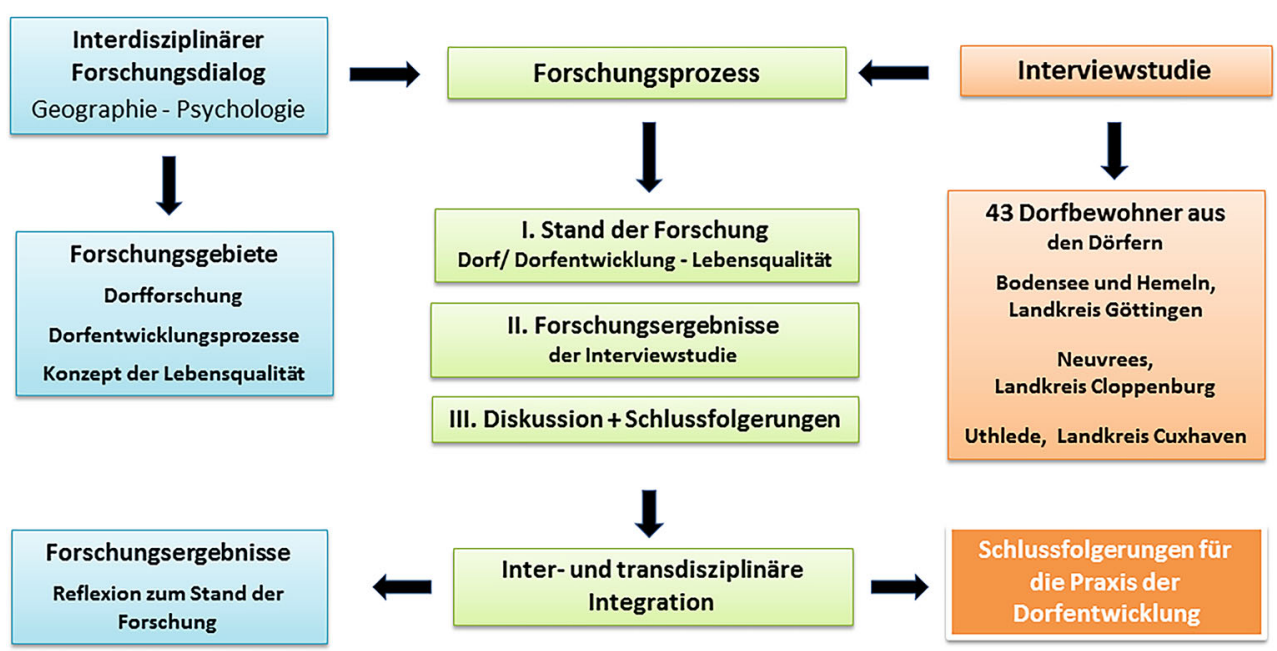

\section{Einordnung in die aktuelle Forschung}

\subsection{Das Dorf: Persistenz und Wandel}

Die charakteristische Siedlungseinheit ländlicher Räume ist das Dorf. Nicht nur die Anzahl der Dörfer in Deutschland ist beeindruckend - Henkel (2012: 9) spricht von 35.000 Dörfern in Deutschland -, sondern auch die Vielfalt der Dörfer fasziniert. Topographische und regionale Lage, Siedlungsform und regionaltypische Baukultur, Urbanisierungsgrad und Verkehrsanbindung prägen diese Vielfalt. Diese ist das Ergebnis eines Prozesses, der „,vom komplexen Zusammenspiel unterschiedlicher externer und interner Faktoren und Prozessregler bestimmt" wird (Born 2011: 10). Trotz hochdynamischer Veränderungen bewahren viele Dörfer bis heute ihre oben beschriebenen typischen Elemente. Troßbach und Zimmermann (2006: 285) sprechen von einem ,grundlegenden Phänomen der Persistenz des Dorfes“, wobei es auch die „gesichtslosen Dörfer“ gibt, die etwa durch Dorfmodernisierungen der 1950er- bis 1970erJahre ihre Individualität weitgehend verloren haben (Henkel 2012: 292). Seit den 1980er-Jahren wird der Bewahrung einer dörflichen Individualität auch mit Bezug zur Lebensqualität im Dorf eine größere Aufmerksamkeit geschenkt, was sich in der Dorfforschung (Hauptmeyer 1983: 211) wie auch in aktuellen Strategiepapieren zur Dorfzukunft widerspiegelt (Niedersächsischer Heimatbund/Niedersächsische Akademie Ländlicher Raum 2014).

Ausgehend von dieser Erkenntnis gehen wir davon aus, dass es enge Bezüge zwischen den Ausprägungen einer dörflichen Individualität und der individuellen Lebensqualität gibt. So zielten die Fragen im Interview (vgl. Kapitel 3.2) unter anderem auf die dörfliche Wohnsituation, die dorfspezifischen Gelegenheiten der Freizeitgestaltung und auf Mitwirkungsmöglichkeiten im Rahmen der Dorfentwicklung ab. Die gefundenen Zusammenhänge werden in diesem Beitrag verdeutlicht (vgl. Kapitel 4).

Der Aspekt der Mitwirkung in der Dorfentwicklung gewinnt seit den 1980er-Jahren an Bedeutung. Born (2009: 60) spricht in diesem Zusammenhang von „dorfbezogenen Governancen" und beschreibt die Entstehung von neuen Dorfvereinen, deren „Handlungsfelder sich nicht mehr nur auf ein spezifisches Aktivitätsfeld, sondern auf das gesamte Dorf und seine Bewohner beziehen“. Die Entwicklung von neuen Dorfvereinen stellt nach Born (2009: 60) eine Reaktion auf infrastrukturelle und demographische Veränderungsprozesse dar, bietet darüber hinaus aber möglicherweise auch dem einzelnen Dorfbewohner eine neue „Selbstwirksamkeitserfahrung", die wiederum zur Steigerung der individuellen Lebensqualität im Dorf beitragen kann (vgl. Kapitel 2.3). Diese Erkenntnis und der von Born (2009: 58) beschriebene Bedeutungszuwachs von Akteurnetzwerken in dörflichen Anpassungs- und Revitalisierungsprozessen können in anderer Form seit den 1990er-Jahren auch in der Dorferneuerungsplanung beobachtet werden. Auf die Entwicklung der Dorferneuerung als dem klassischen staatlichen Instrument der Dorfentwicklung soll in der Folge ein Blick geworfen werden, wobei dem Bedeutungszuwachs der Bürgerbeteiligung in unserem Forschungskontext die besondere Aufmerksamkeit gilt.

\subsection{Dorferneuerung und bürgerschaftliches Engagement}

Die Verantwortung, die der Staat für die Sicherung der Lebensqualität in den Dörfern übernimmt, spiegelt sich in be- 
sonderer Weise in der Entwicklung der Dorferneuerungsplanung wider. Deren Leitbilder und Ziele waren immer eng an die gesellschaftlichen Rahmenbedingungen und Veränderungsprozesse gekoppelt und haben sich dementsprechend in den letzten 70 Jahren erheblich verändert.

In der Nachkriegszeit standen zunächst der Wiederaufbau und die Modernisierung der technischen Infrastruktur im Mittelpunkt. Es folgte eine Phase (1960er- und 1970erJahre) der Dorfsanierung nach städtischen Standards. Henkel (2012: 292) beschreibt die Dorferneuerung dieser Zeit als „eine Phase des unbekümmerten [...] Umgangs mit der ländlichen Baukultur“. Entsprechend des Leitbilds des ,autogerechten Dorfes“" wurden Durchgangsstraßen ausgebaut und oft musste die historische Bausubstanz des Ortskernes einem überdimensionierten Straßenbau weichen. Die mit Flächensanierungen und dem Aufbau von Neubauvierteln verbundene Modernisierung des Dorfes erschien vielen Dorfbewohnern zeitgemäß, denn so konnten sie sich ,einen Traum erfüllen' und ein neues Haus im Dorf, angebunden an ein autogerechtes Straßennetz, beziehen. Diese Entwicklung wurde von vielen Bewohnern zur damaligen Zeit als eine Verbesserung ihrer Lebensqualität bewertet (Henkel 2012: 292).

Die Wiederentdeckung und Wertschätzung der dörflichen Kulturlandschaft mit seiner historischen Baukultur, den typischen Dorfgärten und der das Dorf umgebenden Flur prägt die Dorferneuerungsplanung seit den 1980erJahren. Entscheidend für ihre Weiterentwicklung war unter anderem die Novellierung des Flurbereinigungsgesetzes von 1976. Hierin wurde die bestandsorientierte, erhaltende Dorferneuerung gesetzlich verankert, womit diese sich erstmals offiziell von der Städtebauförderung abhob (Magel/ Bock 2007: 139). Die Dorferneuerungsplanung entwickelte sich zu einem integrierten Planungsinstrument, welches bauliche, agrar- und infrastrukturelle und zunehmend auch ökologische und soziale Aspekte des Dorfes einbezogen hat.

Seit den 1990er-Jahren setzte die Dorferneuerung, anfangs eine Expertenplanung, immer stärker auf die Mitwirkung der Dorfbewohner (Henkel 2012: 295). Magel (2000: 135) hebt hervor: „Die nun allerorten geforderte partizipative Bürgerbeteiligung plus Bürgerverantwortung ist in der Dorferneuerung längst selbstverständlich geworden." Betont wird die Schlüsselrolle der Kommunikation. Die Dorferneuerung hat also schon früh die zivilgesellschaftliche Kraft der Dorfgemeinschaft erkannt und sehr bewusst die Dorfbewohner in die Planung mit einbezogen (Karweik 2009: 29). Beteiligungsprozesse sind in der Dorferneuerungsplanung offenbar erprobt, und dennoch stellt sich die Frage, ob die aktuelle Schrumpfung und Alterung vieler Dörfer verbunden mit dem Verlust von Strukturen der technischen und sozialen Infrastruktur und damit auch der Gefährdung der Lebensqualität nicht neue Formen einer ,lokalen Verantwortungsgemeinschaft“ erfordert. Magel (2011: 123) wählt den Begriff „,lokale Verantwortungsgemeinschaft" und unterstreicht damit, dass es in der Dorfentwicklung zukünftig nicht ausreicht, Bürger zu beteiligen, sondern dass es vielmehr wichtig ist, ihnen im Dorfentwicklungsprozess eine konkrete Verantwortung, etwa im Rahmen einzelner Projekte, zu übergeben. Bezogen auf diese Fragestellung lieferte das Forschungsprojekt „Dorferneuerung 2020 - Zukunftskonzeption und -strategien der Dorferneuerung in Bayern" wichtige Erkenntnisse (Ritzinger 2011: 81 ff.). Besonders interessant in Bezug auf die in diesem Beitrag dargestellte Forschung ist die Erkenntnis, dass ,dem Ausbau von Beteiligungsstrukturen, die über das (Dorferneuerungs-) Verfahren hinausgehen, eine immer größere Bedeutung zukommt“" (Ritzinger 2011: 87 f.). In Niedersachsen wurden in einem Modellprojekt weitergehende Formen der Bürgerbeteiligung in der Dorferneuerungsplanung bereits erprobt (Karweik 2009: 33). Eine Erkenntnis aus diesem Modellprojekt formuliert Karweik (2009: 29) wie folgt: „Fest steht [...], dass die Dorfbewohner als Partner von Planern und Gemeinden fit gemacht werden [sollten], um bewusster agieren und von Beginn an besser durchstarten zu können“. Auch Ritzinger (2011: 88) sieht Qualifizierungsbedarf und die Notwendigkeit, dass eine Weiterentwicklung der Beteiligungsmethoden im Sinne eines Kapazitätsaufbaus („Capacity Buildings“) aller Akteure stattfindet, und leitet daraus eine neue Aufgabe für die „Schulen der Dorf- und Landentwicklung“ in Bayern ab. Da es in Niedersachsen keine Schulen der Dorfund Landentwicklung gibt, werden andere Wege der Qualifizierung erprobt. So wurde beispielsweise vom Verein „Freie Altenarbeit Göttingen e. V. - mobile wohnberatung südniedersachsen“ in Kooperation mit dem LEADERRegionalmanagement Göttinger Land eine Fortbildung zum Thema Dorfmoderation entwickelt und in den Jahren 2012-2013 erstmals angeboten. Diese soll Kenntnisse und Kompetenzen für die Unterstützung oder Initiierung von Prozessen in einzelnen Dörfern, die zur Förderung der Lebensqualität im ländlichen Raum beitragen, vermitteln. Dies stellt eine Möglichkeit dar, Dorfbewohner für eine Verantwortungsübernahme in der Dorfentwicklung zu qualifizieren. Eine Diskussion verschiedener Formen der Unterstützung von Dörfern zur Selbsthilfe, einschließlich des Ansatzes der Dorfmoderation, findet sich bei EignerThiel/Meyer (2015).

Insgesamt ist die Bereitschaft, sich zu engagieren, in der Bevölkerung heute sehr hoch: Der Freiwilligensurvey 2009 ergab, dass 36 Prozent der deutschen Bevölkerung bürgerschaftlich engagiert sind, und zwar mit steigender Tendenz. Mit dem Engagement wollen sie unter anderem etwas zum Gemeinwesen beitragen: „Trotz der heute bei vielen Menschen vorhandenen Politikverdrossenheit engagieren sich die allermeisten Menschen [...] deswegen, weil 
sie die Gesellschaft (zumindest im Kleinen) mitgestalten wollen“ (Gensicke/Geiss 2010: 12). Auch das Institut für Demoskopie Allensbach (BMFSFJ 2013: 3) konnte als Motiv für bürgerschaftliches Engagement feststellen, dass 70 Prozent der Befragten damit das „Leben am Wohnort attraktiver machen“ möchten. Bedeutend ist, dass freiwilliges Engagement auf dem Land stärker verbreitet ist als in größeren Städten, eventuell gerade deshalb, weil hier weniger Angebote verfügbar sind und so aus eigener Kraft die Lebensqualität ein Stück weit aufrechterhalten werden kann (Gensicke/Geiss 2010: 26; vgl. Kapitel 2.3). Dabei wird vor allem den Vereinen eine hohe Bedeutung zugesprochen, was wiederum die Verbindung zu den von Born (2009: 60) beschriebenen „neuen Dorfvereinen“ herstellt.

Die aktuelle Forschung zur Dorfentwicklung und zum bürgerschaftlichen Engagement zeigt, dass es bereits innovative Ansätze zur Weiterentwicklung der Dorferneuerung wie auch neue methodische Ansätze der Dorfentwicklung gibt. Zusätzlich ist die Bereitschaft, sich zu engagieren, gerade in ländlichen Räumen hoch. Aber sind diese Ansätze geeignet, um den schleichenden Verlust an Lebensqualität in den Dörfern zu bremsen und knüpfen sie an die Bedürfnisse der Dorfbewohner an? Diese Fragen wurden im Kontext des Forschungsvorhabens „Lebensqualität im Dorf" diskutiert und am Ende dieses Beitrags werden hierauf erste Antworten gegeben.

\subsection{Lebensqualität in Dörfern}

Grundsätzlich ist Lebensqualität ein Konzept, das sowohl ,materielle wie auch immaterielle, objektive und subjektive, individuelle und kollektive Komponenten umfasst und das ,Besser' gegenüber dem ,Mehr' betont“" (Noll 2000: 3). Zum einen sind es die faktischen Lebensbedingungen (z. B. Größe des Wohnplatzes, Vorhandensein eines Gartens, Lage des Hauses, Verkehrsinfrastruktur, Versorgung mit Lebensmitteln, Ärzten, Bildung, Kultur), zum anderen die individuellen Ressourcen der Bürger (z. B. soziale Beziehungen, Intelligenz, familiäre Situation), die das Lebensgefühl ausmachen. Zu den subjektiven Aspekten von Lebensqualität gehört die persönliche Bewertung der eigenen Lebenslage: Gefühle wie Zufriedenheit und Glück oder auch Besorgnisse und Einsamkeit (Ebbinghaus/Noll/Bahle et al. 2006: 5).

Eine hohe Lebensqualität wird demnach nur erreicht, wenn sowohl objektive wie auch subjektive Faktoren positiv ausgeprägt sind - hoher materieller Wohlstand reicht nicht aus, um hohe Zufriedenheit zu erlangen; andererseits ist ein gewisser Wohlstand nötig, um zufrieden sein zu können. Hunecke (2013: 56) resümiert: „Nur wenn es gelingt, die subjektive Sichtweise der betroffenen Menschen mit zu erfassen, kann die Lebensqualität einer Stadt, einer Region oder auch von ganzen Nationen im Sinne einer Sozialberichterstattung angemessen beurteilt werden.“

$\mathrm{Zu}$ einer guten Lebensqualität gehört auch eine hohe Selbstwirksamkeitsüberzeugung im Sinne von Bandura (1991: 248) und Schwarzer (2004: 12 ff.). Diese ist definiert als die Erwartung, dass das eigene Handeln und die eigenen Kompetenzen ausreichen, um komplexe Anforderungssituationen bewältigen zu können. Auf den Kontext des Dorfes bezogen bedeutet dies: Die Erfahrung, seine Wünsche und Vorstellungen bei der Gestaltung des Dorflebens erfolgreich einbringen zu können, kann sich zukünftig positiv auf die Motivation auswirken, sich weiter an der Dorfentwicklung zu beteiligen. Bezieht sich diese Überzeugung auf gemeinschaftliches Handeln, ist man also der Auffassung, man könne vor allem durch das Zusammenwirken einer Gruppe erfolgreich sein, spricht man von der „kollektiven Selbstwirksamkeitserwartung“ (Schmitz/Schwarzer 2002: 195). Selbstwirksamkeitserfahrungen können die Menschen insgesamt mit dem Gefühl erfüllen, etwas Sinnvolles zu tun, was sich auf die subjektive Lebensqualität wiederum positiv auswirkt. Eine Förderung der Selbstwirksamkeit stärkt letztlich politisches Empowerment (Herriger 2006: 14 f.; 197 ff.) in Richtung auf die Entwicklung von Maßnahmen und Initiativen zur Förderung eines sozialen und kulturellen Wandels, wie er heute in den Dörfern ansteht. Zahlreiche empirische Studien untermauern den Effekt, dass ehrenamtliches Engagement Aspekte von Lebensqualität, insbesondere die Selbstwirksamkeitsüberzeugung, verbessern kann (Legewie/Janßen 1997: 351 ff.; Eigner-Thiel 2005: 210 ff.). Diese kann wiederum die psychische Gesundheit langfristig positiv beeinflussen (Bengel/Strittmatter/Willmann 2001: 54).

Sucht man gezielt nach Forschungsvorhaben im Bereich „Lebensqualität von Dörfern“, findet man wenige empirische Erhebungen zu diesem Thema. Forschungsinstitutionen wie das Berlin-Institut für Bevölkerung und Entwicklung (2015) befassen sich zwar mit einzelnen Aspekten der Daseinsvorsorge im ländlichen Raum, aber nicht explizit mit der Lebensqualität als Ganzem und auch nicht aus der Perspektive von Dorfbewohnern selbst. Es sind nur wenige Ansätze erkennbar, in denen die Themen „Lebensqualität“ und „Dorf“" explizit kombiniert wurden wie beispielsweise in einem Workshop im Land Brandenburg. Becker (2012: 10 f.) stellte dort zum Thema „Lebensqualität in Dörfern und Städten“ auch die subjektiven Bedürfnisse der Menschen in den Vordergrund. Er spricht sich bezüglich der Erhaltung der dörflichen Lebensqualität für eine Nutzung der Fähigkeiten der Akteure vor Ort aus und stellt dazu die Hypothese auf, dass dieses bürgerschaftliche Engagement auch Spaß macht - ein Nachweis dafür bleibt aber offen.

Kunz und Schumacher (2010: 54; 65) stellen fest, dass sich objektive Gegebenheiten wie Nahversorgung, Bildung und Gesundheitseinrichtungen in großem Maße auch auf 
Abb. 2: Lage der in die Studie einbezogenen Dörfer in Niedersachsen

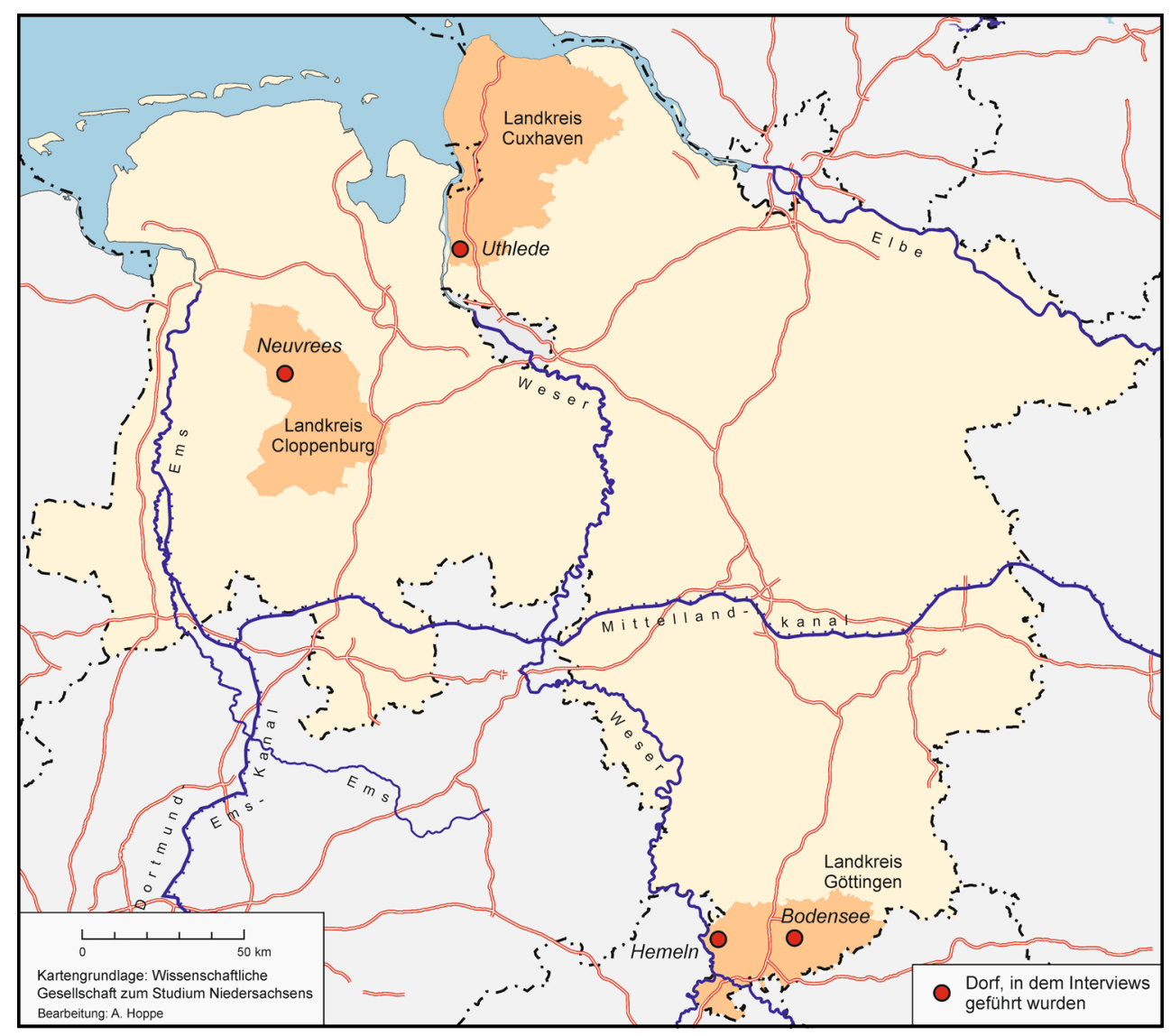

die individuelle subjektive Lebensqualität in Dörfern auswirken können. Sie sehen Möglichkeiten zur Erhöhung der Lebensqualität auf dem Lande durchaus darin, dass lokale Akteure durch ihr Engagement fehlende Infrastruktur kompensieren, aber ihr Ansatz ist eher normativ als empirisch fundiert.

Eine aussagekräftige quantitative Studie zur Bewertung der Lebensverhältnisse und Daseinsvorsorge aus der Sicht der Bewohner wurde von Freitag, Rößler und Kunz (2009) in 22 sächsischen Dörfern durchgeführt. Das Ergebnis zeigte, dass 60 Prozent der Bewohner zufrieden oder sehr zufrieden und nur 6 Prozent unzufrieden oder sehr unzufrieden mit den Lebensverhältnissen waren (Freitag/Rößler/Kunz 2009: 3). Als statistisch relevante Einflussfaktoren zeigten sich unter anderem die beiden Aspekte „Zusammenhalt im Dorf“ und „Wohnumfeld und Wohnverhältnisse“. Offen bleibt hier, was genau diese beiden Aspekte so attraktiv macht, wie die Zusammenhänge und Begründungen dafür aussehen.

Die Auswertung zum Stand der Forschung zeigt weiter, dass bei der Erhebung statistischer Daten in der Bevölkerung - und dies gilt auch für subjektive Bewertungen objektiver Aspekte - meist zwischen Bewohnern aus West- und Ostdeutschland differenziert wird, fast nie jedoch zwischen Stadt- und Landbewohnern. Eine der wenigen Ausnahmen bilden Erhebungen von Habich und Spellerberg (2013: 310 ff.): Hier werden Zufriedenheitsbewertungen bezüglich ausgewählter Lebensbedingungen nach fünf Raumtypen (von der Großstadt bis zur Landgemeinde) dargestellt. Es zeigt sich, dass in Westdeutschland 2011 in der kleinsten Wohneinheit, der Landgemeinde, drei von vier Zufriedenheitswerte (Zufriedenheit mit dem Leben, mit der Gemeinde, mit der Wohnumgebung) am höchsten waren (Spellerberg/ Huschka/Habich 2006: 12; Habich/Spellerberg 2013: 321).

Zusammenfassend zum Stand der Forschung lässt sich konstatieren, dass es zwar einige quantitative Studien gibt, in denen der Zusammenhang zwischen Lebensqualität und Dorfentwicklung thematisiert wird; es scheint diesbezüglich hingegen keine qualitativen Studien zu geben, die die hinter den Befunden liegenden Zusammenhänge aufzeigen könnten.

\section{Das Forschungsvorhaben ,Lebensqualität im Dorf"6}

\subsection{Ziel}

Im Mittelpunkt der Untersuchung stand der Bereich des „Lebens im Dorf“, der in Bezug auf objektive Faktoren wie 
Wohnlage, Nahversorgung, Bildungsmöglichkeiten, medizinische und kulturelle Versorgung etc. erfasst wurde. Zum einen sollte das objektive Vorhandensein erhoben werden, zum anderen die subjektive Bewertung seitens einzelner Dorfbewohner beschrieben und analysiert werden. Auch die Selbstwirksamkeitserwartung in Bezug auf politische Gemeinwesenarbeit sollte in den Interviews eruiert werden.

\subsection{Methodisches Vorgehen}

\subsubsection{Kriterien für die Auswahl der Dörfer}

Begonnen wurde die Erhebung im April 2013 in zwei Dörfern im Landkreis Göttingen in Südniedersachsen. 2014 wurde sie in den Landkreisen Cuxhaven und Cloppenburg, beide ebenfalls in Niedersachsen, fortgeführt. Die Dörfer mussten mehrere Kriterien erfüllen: Sie sollten zum einen etwa 800 bis 1.000 Einwohner haben. Dahinter steckt die Annahme, dass solche Dörfer groß genug sind, um eine gewisse Infrastruktur, beispielsweise einen Dorfladen, einen Kindergarten und eventuell auch noch eine Grundschule, vorzuhalten und ein ausreichendes Ausmaß an dörflicher Aktivität zu bieten. Andererseits sind sie klein genug, um über eine ausreichende Zahl von Netzwerken - das heißt mindestens noch oder wieder über einige Vereine oder andere Gruppierungen wie Ortsrat und/oder Kirchenrat - zu verfügen, sodass sich Ideen und Geschehnisse im Ort leicht herumsprechen und die Dorfgemeinschaft sich gegenseitig genügend kennt und kaum Anonymität herrscht.

Ein weiteres Auswahlkriterium war die relativ periphere Lage innerhalb der Landkreise: Gesucht waren Dörfer, die mindestens 20 Kilometer vom nächsten Oberzentrum entfernt liegen. Wichtig war weiterhin, dass die Dörfer unterschiedlich vom demographischen und wirtschaftsstrukturellen Wandel betroffen waren. Außerdem sollten Dörfer aus verschiedenen Kulturlandschaftsräumen mit unterschiedlichen Traditionen in die Untersuchung einbezogen werden. Die Kontaktaufnahme in den Dörfern erfolgte über den Ortsvorsteher und den Gemeinderat. Alle Dörfer waren ohne Bedenken bereit, an der Studie teilzunehmen.

\subsubsection{Auswahl der Untersuchungsregionen und Dörfer}

Für die Studie wurden im Landkreis Göttingen die Dörfer Hemeln und Bodensee, im Landkreis Cuxhaven das Dorf Uthlede und im Landkreis Cloppenburg das Dorf Neuvrees ausgewählt (vgl. Abbildung 2).

In Tabelle 1 sind wichtige Merkmale der Dörfer zusammengestellt.

\subsubsection{Auswahl der Interviewpartner}

In den Dörfern im Landkreis Göttingen wurden persönliche Gespräche mit den Ortsräten aus Hemeln und Bodensee geführt, um die Forschungsziele vorzustellen und die Dörfer zu gewinnen. Beide Dörfer zeigten Interesse an der Teilnahme. In den Dörfern Uthlede (Landkreis Cuxhaven) und Neuvrees (Landkreis Cloppenburg) wurde die Studie telefonisch vorgestellt; das Interesse zur Teilnahme war hier ebenfalls vorhanden. Die Ortsräte stellten dann auf Bitte der Studienleiter Listen von möglichen Interviewpartnern zusammen. Diese Listen enthielten jeweils ältere und jüngere Gesprächsteilnehmer, Einheimische und Zugezogene, engagierte (in Vereinen, Ortsrat, Kirche) und nichtengagier-

Tab. 1: Vergleichende Darstellung der vier Untersuchungsdörfer

\begin{tabular}{|c|c|c|c|c|}
\hline Merkmale/Dorf & Hemeln & Bodensee & Uthlede & Neuvrees \\
\hline Landkreis & Göttingen & Göttingen & Cuxhaven & Cloppenburg \\
\hline Region & Südniedersachsen & Südniedersachsen & Nordniedersachsen & Nordwestnieder-sachsen \\
\hline Einwohnerzahl $2013^{a}$ & 972 & 879 & 992 & 939 \\
\hline $\begin{array}{l}\text { Siedlungstyp, } \\
\text { Alter des Dorfes }\end{array}$ & $\begin{array}{l}\text { Haufendorf, } \\
\text { ca. } 1.100 \text { Jahre }\end{array}$ & $\begin{array}{l}\text { Haufendorf, } \\
\text { ca. } 900 \text { Jahre }\end{array}$ & $\begin{array}{l}\text { Straßendorf, } \\
\text { ca. } 260 \text { Jahre }\end{array}$ & $\begin{array}{l}\text { Straßendorf, } \\
\text { ca. } 225 \text { Jahre }\end{array}$ \\
\hline $\begin{array}{l}\text { Entfernung zum } \\
\text { Oberzentrum }^{\mathrm{b}}\end{array}$ & $\begin{array}{l}28 \text { km (Göttingen) } \\
36 \text { Autominuten }\end{array}$ & $\begin{array}{l}21 \mathrm{~km} \text { (Göttingen) } \\
25 \text { Autominuten }\end{array}$ & $\begin{array}{l}35 \mathrm{~km} \text { (Bremen) } \\
24 \text { Autominuten }\end{array}$ & $\begin{array}{l}41 \mathrm{~km} \text { (Oldenburg) } \\
50 \text { Autominuten }\end{array}$ \\
\hline Anzahl Vereine & 21 & 9 & 12 & 17 \\
\hline Dorfgemeinschaftshäuser & Dreschschuppen & $\begin{array}{l}\text { DGH I, DGH II, } \\
1 \text { Jugendraum }\end{array}$ & $\begin{array}{l}\text { Heimathaus } \\
\text { Sporthaus }\end{array}$ & $1 \mathrm{DGH}$ \\
\hline Gastronomie & 1 Pension, 1 Gasthaus & keine & $\begin{array}{l}1 \text { Gasthaus, } \\
1 \text { Pizzeria }\end{array}$ & keine \\
\hline Grundschule & ja & mit Nachbardorf & ja & ja \\
\hline Kindergarten & ja & mit Nachbardorf & ja & mit Nachbardorf \\
\hline neuartige Dorfvereine & $\begin{array}{l}\text { thematische Arbeits- } \\
\text { gruppen und Dorfmo- } \\
\text { deration }\end{array}$ & $\begin{array}{l}\text { „Zukunftsrunde Bo- } \\
\text { densee“ }\end{array}$ & „Runder Tisch Uthlede“ & $\begin{array}{l}\text { Verein „Die Dorfge- } \\
\text { meinschaft““ }\end{array}$ \\
\hline
\end{tabular}

${ }^{a}$ Mündliche Auskunft der jeweiligen Ortsbürgermeister oder Ortsvorsteher zum Zeitpunkt der Untersuchung

${ }^{\mathrm{b}}$ Entfernungsmessung mit google maps 
te Dorfbewohner, um möglichst heterogene Meinungen abbilden zu können. Alle vorgeschlagenen Personen zeigten sich bereit, an den Interviews teilzunehmen. 2013 wurden in Hemeln zwölf, in Bodensee zehn Personen interviewt. In Uthlede wurden 2014 zehn und in Neuvrees elf Einwohner interviewt.

\subsubsection{Problemzentriertes Interview}

Zur Untersuchung der beschriebenen Fragen nach der Lebensqualität und dahinterliegenden Zusammenhängen in Dörfern eignen sich qualitative Methoden besonders. Bei qualitativen Interviews werden einander ähnliche Einzelfälle in die Tiefe gehend beschrieben, um induktiv auf weitere Fälle zu schließen. Sie zeichnen sich durch Anschaulichkeit und Lebensnähe aus, die bei komplexen Sachverhalten wie dem demographischen Wandel eine Rolle spielen. Hintergründe und Zusammenhänge können aufgedeckt werden, was bei quantitativ auszuwertenden Fragebögen nicht der Fall ist. Im vorliegenden Beispiel wurde das problemzentrierte Interview nach Witzel (2000) verwendet. Es lehnt sich weitgehend an das theoriegenerierende Verfahren der „Grounded Theory“ (Glaser/Strauss 1998) an. Im Forschungsprozess wird dabei sowohl bei der Datenerhebung als auch bei der Datenauswertung im Wechsel induktiv und deduktiv vorgegangen. Das problemzentrierte Interview hat somit keinen rein explorativen Charakter, sondern ist auch theoriegeleitet, da die Aspekte vorangegangener Problemanalysen in die Gestaltung des Interviewleitfadens einfließen. Damit bot sich diese Methode für die vorliegende Fragestellung besonders an.

\subsubsection{Interviewleitfaden}

Im Interviewleitfaden wurden die folgenden Aspekte als operationalisierte Komponenten von Lebensqualität erhoben:

- Wohndauer

- Lebensumfeld (Wohnlage, Alltag, Mobilität)

- soziales Miteinander (Dorfgemeinschaft, Vereine)

- Selbstverwirklichung und Akzeptanz im Dorf

- Lebenszufriedenheit (Dorf, Stadt)

- Dorfentwicklung und demographischer Wandel

- Sonstiges

Nach einer einführenden Vorstellung des Kontexts der Studie wurden die Bereiche nacheinander durchgegangen. Es gab jeweils objektive und subjektive Fragekomponenten, zu denen Nachfassfragen und nähere Erläuterungen vorbereitet waren.

\subsubsection{Durchführung der Interviews}

Das problemzentrierte Interview ist ein diskursiv-dialogisches Verfahren. Dabei werden in diesem Fall die Dorfbewohner als Experten ihrer Orientierungen und Handlungen begriffen, die im Gespräch ihre Sichtweisen verdeutlichen und dabei auch eigene Aussagen oder die der Interviewer korrigieren können. Wichtig ist deshalb bei der Interviewdurchführung das „Prinzip der Offenheit“, was bedeutet, dass der Interviewte ohne vorgegebene Antwortalternativen frei antworten kann (vgl. Mayring 2002). Die Interviews fanden überwiegend bei den Dorfbewohnern zu Hause im gewohnten Umfeld und nur in Einzelfällen auch an deren Arbeitsplatz oder an der Hochschule in Göttingen statt und dauerten im Schnitt rund 1,25 Stunden. Sie wurden aufgezeichnet und anschließend wörtlich transkribiert.

\subsubsection{Auswertungsmethode}

Die Auswertungsmethode lehnt sich an Prinzipien der Grounded Theory nach Strauss und Corbin (1990) an. Grundlage der Auswertung sind transkribierte Interviews, die einzeln Satz für Satz analysiert und abschnittsweise markiert werden. Erste Ergebnisse dieses Interpretationsschrittes sind jeweils fallspezifisch zentrale Themen, die $\mathrm{zu}$ einer Aussage verdichtet werden. Es folgt ein systematischer Vergleich der Interviews mit dem Ziel, fallübergreifende Kategorien herauszuarbeiten. Ziel ist es dabei, sogenannte Kernkategorien zum Beispiel in Form eines Typologiekonzepts zu entwickeln, die in der nächsten Auswertungsstufe mit weiterem empirischem Material gestützt werden.

\section{Ergebnisse}

\subsection{Besonderheiten der Lebensqualität in den Dörfern aus der Sicht ihrer Bewohner}

Im Folgenden werden zunächst die von den Befragten geschilderten Charakteristika der dörflichen Lebensqualität beschrieben und Schlussfolgerungen für Dorfentwicklungsprozesse abgeleitet. Anschließend werden Ergebnisse zu den vorgestellten Themenschwerpunkten dargestellt.

\subsubsection{Die Dörfer im Landkreis Göttingen}

Hemeln: In Hemeln mit seinen alten kleinbäuerlichen Strukturen herrscht laut Aussagen der Interviewpartner eine angenehme Mischung aus „Ruhe“ und „,vielen Angeboten" vor. Außerdem werden ein ,guter Zusammenhalt" und ein „hohes Wir-Gefühl“ genannt. Damit verbunden ist die Tatsache, dass sehr viele Vereine miteinander kooperie- 
ren statt zu konkurrieren. Ebenso wird die Zusammenarbeit des Ortsrates dargestellt: Auch dieser ziehe meist an einem Strang in Richtung der Belange des Dorfes. Als Gründe für den guten Zusammenhalt werden zwei Aspekte genannt: Aktive Vereinstätigkeit wird von den Eltern vorgelebt und viele Menschen sind seit Jahrhunderten im Dorf verwurzelt und setzen sich deshalb gerne dafür ein. Unterstützt werden die Dorfprozesse von einer im Ort ansässigen Dorfmoderatorin. Erfolgreich durchgeführte gemeinschaftliche Unternehmungen sind beispielsweise der Erhalt der Grundschule oder der Umbau eines alten Dreschschuppens zum Dorfgemeinschaftshaus.

Bodensee: Was Bodensee aus Sicht der Befragten besonders auszeichnet, ist die hohe Toleranz und Offenheit seiner Bewohner. Diese zeigt sich im unkomplizierten Umgang mit Zugezogenen und auch mit Homosexuellen oder Behinderten, die gut ins Dorf integriert sind. Zurückgeführt wird diese Toleranz auf Erfahrungen mit Flüchtlingen, die die Dorfbewohner nach dem Krieg gemacht haben: Damals haben die Landwirte Teile ihrer Flächen für ein großes Neubaugebiet im Dorf zur Verfügung gestellt, sodass die Bodenseer bis heute an neue Gesichter im Dorf gewöhnt sind. Als weitere Besonderheit werden die noch gut erhaltene Infrastruktur (z. B. mit ,Ringschule“: zwei Jahre in Bodensee, zwei Jahre im Nachbardorf; Kindergarten; Krippe) und die Dorfgemeinschaftshäuser hervorgehoben, weil sich die Menschen hier beispielsweise zu Feierlichkeiten treffen können. Auch Personen aus den Nachbardörfern mieten sich diese Häuser. Eine Besonderheit in Bodensee ist außerdem die sogenannte Zukunftsrunde: ein Gremium aus Bürgern, das die Belange des Dorfes tatkräftig mit unterstützt, indem es Veranstaltungen organisiert und neue Ideen umsetzt.

\subsubsection{Das Dorf Uthlede im Landkreis Cuxhaven}

Als Besonderheit des Dorfes wird an erster Stelle die gute soziale Infrastruktur genannt. Dazu zählt vor allem eine Schule, zu der auch viele Kinder aus den Nachbardörfern kommen. Aber auch eine Sporthalle, ein Heimathaus und eine Waffelfabrik werden besonders gelobt. Uthlede wird ebenfalls als aktive und starke Dorfgemeinschaft beschrieben, die die gute Infrastruktur als solide Grundlage nutzen kann, sie andererseits aber auch selbst mit erhält und mit geschaffen hat. Uthlede gehört seit Beginn des Jahres 2014 zur Einheitsgemeinde Hagen und hat damit seine Eigenständigkeit verloren. Hier ist den Befragten noch unklar, was das für Folgen für das Dorfleben haben wird. In der Konsequenz hat sich der „Runde Tisch Uthlede“ gegründet, der aus interessierten Vereinsvorständen besteht, die sich gemeinsam mit der Ortsvorsteherin um die Belange des Dorfes kümmern. Der Dorfgemeinschaft werden eine ,hohe Offenheit“ und ein ,guter Zusammenhalt“" bescheinigt, zum anderen wird explizit eine große Kinderfreundlichkeit beschrieben, die sich unter anderem in vielen Angeboten für diese zeigt.

\subsubsection{Das Dorf Neuvrees im Landkreis Cloppenburg}

Neuvrees war ursprünglich ein Bauerndorf und ist heute immer noch ein landwirtschaftlich geprägtes Straßendorf. Der angrenzende Eleonorenwald wird von den Bewohnern gerne zur Naherholung genutzt. Neben landwirtschaftlichen Betrieben befinden sich im Dorf mehrere Gewerbe- und Handwerksbetriebe. Seit der Gebietsreform 1974 ist Neuvrees Mitglied der Einheitsgemeinde Friesoythe. Im Dorf gibt es deshalb auch keinen eigenständigen Gemeinderat, sondern nur einen Ortsvorsteher. Zur Unterstützung wurde der Verein „Die Dorfgemeinschaft“" mit 18 Personen gegründet, die gemeinsam mit dem Ortsvorsteher Veranstaltungen auf die Beine stellen. Mindestens einmal im Jahr finden öffentliche Bürgerversammlungen statt. Das Dorf Neuvrees geht in das Dorf Gehlenberg über. Viele Vereine sind dorfübergreifend organisiert. Es gibt in Gehlenberg einen Laden und einen Arzt. Als Besonderheit von Neuvrees wird von den Befragten vor allem der hohe Zusammenhalt der Menschen genannt. Festgemacht wird dies an der fast geschlossenen Teilnahme an Festen (z. B. am Schützenfest), die die Bewohner gemeinsam organisieren und für die sich die meisten extra Urlaub nehmen. Außerdem existierten eine hohe gegenseitige Unterstützung untereinander, weil das Dorf ,,nicht zu groß, sondern wie eine große Familie" sei. In Neuvrees ist es außerdem typisch, dass die Eltern mit im Haus oder im Nachbarhaus leben und Eltern ihren Kindern ein Grundstück überlassen. Kinder pflegen die Eltern, Großeltern hüten die Enkel. Befragte sprechen von einem freiwilligen „Generationenvertrag“, der in Neuvrees unausgesprochen existiere. Typisch für Neuvrees sei außerdem, dass die Menschen meist nicht freiwillig wegziehen. Im Ort gibt es im Vergleich zu anderen Dörfern in Niedersachsen noch relativ viele Arbeitsplätze, da neben den landwirtschaftlichen Betrieben auch mehrere Handwerksbetriebe und Dienstleister ansässig sind.

\subsection{Faktoren für eine gute Lebensqualität in Dörfern und deren Bedeutung für Dorfentwicklungsprozesse}

Aus den durch die Interviewten geschilderten Besonderheiten der Dörfer lassen sich Faktoren ableiten, die eine gute Lebensqualität mit ausmachen. Diese werden in Tabelle 2 in der linken Spalte aufgelistet. In der mittleren Spalte werden den Faktoren Wirkungen auf das Dorfleben zugeordnet. Rechts werden Schlussfolgerungen bzw. Empfehlungen für Dorfentwicklungsprozesse abgeleitet. 
Tab. 2: Faktoren und ihre Bedeutung für Dorfentwicklungsprozesse

\begin{tabular}{|c|c|c|}
\hline $\begin{array}{l}\text { Faktoren für eine gute Lebensqualität } \\
\text { im Dorf }\end{array}$ & Wirkung auf das Dorfleben & $\begin{array}{l}\text { Schlussfolgerungen/Empfehlungen für Dorf- } \\
\text { entwicklungsprozesse }\end{array}$ \\
\hline Kooperation von Vereinen & Erhalt eines vielfältigen Angebots & - Thematisierung des Vereinslebens \\
\hline $\begin{array}{l}\text { Vorgelebtes ehrenamtliches Engagement } \\
\text { der Eltern }\end{array}$ & Stärkung des Ehrenamts & - Würdigung des Ehrenamts \\
\hline Spaß am Engagement & $\begin{array}{l}\text { Stabilisierung und Motivation für weiteres } \\
\text { Engagement }\end{array}$ & - Ehrenamt im Prozess verankern \\
\hline Toleranz, Offenheit & Erleichterung von Integration und Zuzug & $\begin{array}{l}\text { - Willkommenskultur als Thema der Dorfent- } \\
\text { wicklung }\end{array}$ \\
\hline Vorhandene Treffpunkte (Gebäude) & $\begin{array}{l}\text { Durchführung gemeinschaftlicher Planungen } \\
\text { und Unternehmungen }\end{array}$ & $\begin{array}{l}\text { - Instandhaltung und ggf. Neubau von Gebäu- } \\
\text { den }\end{array}$ \\
\hline Grundschule, Kindergarten & $\begin{array}{l}\text { wichtiger Attraktivitätsfaktor insbesondere für } \\
\text { Familien mit Kindern }\end{array}$ & $\begin{array}{l}\text { - Bildung thematisieren; Angebote ggf. dorf- } \\
\text { übergreifend konzipieren }\end{array}$ \\
\hline Zukunftsrunde, Runder Tisch & $\begin{array}{l}\text { Grundlage für eine neue Verantwortungsge- } \\
\text { meinschaft }\end{array}$ & $\begin{array}{l}\text { - neue Dorfvereine in Dorfentwicklungsprozes- } \\
\text { se einbinden }\end{array}$ \\
\hline Naturnähe & $\begin{array}{l}\text { Erholungsmöglichkeit und Umweltbildung } \\
\text { (für Kinder) vor der Haustür }\end{array}$ & $\begin{array}{l}\text { - als wichtiges Thema in der Dorfentwicklung } \\
\text { belassen }\end{array}$ \\
\hline $\begin{array}{l}\text { Regelmäßige öffentliche Bürgerversamm- } \\
\text { lungen }\end{array}$ & $\begin{array}{l}\text { Kommunikation und Transparenz als Schlüs- } \\
\text { sel für eine erfolgreiche Dorfentwicklung }\end{array}$ & $\begin{array}{l}\text { - neue Kommunikationsformate und -methoden } \\
\text { entwickeln }\end{array}$ \\
\hline $\begin{array}{l}\text { Freiwilliger „Generationenvertrag“ (vgl. } \\
\text { Neuvrees) }\end{array}$ & $\begin{array}{l}\text { gegenseitige Unterstützung (Alt hilft Jung } \\
\text { und umgekehrt); Entlastung von Pflege- und } \\
\text { Betreuungsinstitutionen }\end{array}$ & $\begin{array}{l}\text { - Bedingungen und Möglichkeiten diskutieren; } \\
\text { ggf. Innenentwicklung in den Blick nehmen }\end{array}$ \\
\hline Vorhandener Dorfmoderator & $\begin{array}{l}\text { Unterstützung der Selbstorganisation und } \\
\text { Selbstgestaltung im Dorf }\end{array}$ & $\begin{array}{l}\text { - Qualifizierung anbieten und (finanzielle) För- } \\
\text { derung vorsehen }\end{array}$ \\
\hline
\end{tabular}

Zwei Faktoren von besonderer Bedeutung und deren Wirkungen konnten in allen Dörfern abgeleitet werden und werden im Folgenden detaillierter betrachtet.

\subsubsection{Faktor 1: Aktive Dorfgemeinschaften mit „neuen Bürgervereinen“"}

Die untersuchten Dörfer zeichnen sich alle durch ausgesprochen gute Dorfgemeinschaften aus, die jeweils schon viele Projekte gemeinschaftlich erfolgreich gestemmt haben. Diese Dorfgemeinschaften werden genutzt, um sich den heutigen Problemen entgegenzustellen: In allen vier Dörfern wurden zusätzlich zu den vorhandenen (politischen) Strukturen neue Gremien gebildet, wie der „Runde Tisch“ in Uthlede, der Verein ,Die Dorfgemeinschaft" in Neuvrees, die ,Zukunftsrunde“ in Bodensee oder ein komplettes Arbeitsgruppensystem mit einer unterstützenden Dorfmoderatorin in Hemeln. Vorhandene Potenziale die Kompetenzen der Bewohner - werden in diesen Dörfern kreativ genutzt, um das Dorfleben aktiv den eigenen Bedürfnissen anzupassen.

\subsubsection{Faktor 2: Das richtige Maß zwischen „Leben mit Traditionen “ und „Offenheit für Neues “}

In den vier untersuchten Dörfern spielen - gerade beispielsweise bei Festen im Jahresverlauf - auf der einen Seite Traditionen eine große Rolle, die Erfolgsfaktor für das Wohlfühlen und damit auch für das Fortbestehen der Dorfge- meinschaft sind. Auf der anderen Seite werden Offenheit und Toleranz als wichtige Faktoren genannt, die ein Lebendig-Bleiben der Dörfer im Laufe der Zeit ermöglicht haben. Empirisch zeigt sich diese Toleranz beispielsweise in Bodensee. In Hemeln wird Toleranz zwischen dem Bürgermeister und Ortsrat auf der einen sowie einer unabhängigen Dorfmoderatorin auf der anderen Seite geübt. In Neuvrees werden Spätaussiedler integriert und auch aus Uthlede wird eine hohe Offenheit gegenüber Zugezogenen berichtet.

\subsection{Konsequenz des Engagements: Hohe Selbstwirksamkeitsüberzeugung als Potenzial für weitere erfolgreiche Dorfentwicklungsprozesse}

Als Voraussetzung und Folge des bürgerschaftlichen Engagements lassen sich die hohen Selbstwirksamkeitserwartungen der Bürger in den vier Dörfern hervorheben. Die Bewohner meinen mehrheitlich, dass man durch eigene Initiative im eigenen Dorf etwas bewirken, die Dorfgemeinschaft mitgestalten kann. Hier zeigt sich ein positiver Kreislauf: Dadurch, dass die Menschen untereinander sehr vertraut sind, die dörflichen Strukturen gut kennen und die genannte Offenheit vorhanden ist, konnten sie oft die Erfahrung machen, erfolgreich mitgestalten zu können. Dies erhöht ihre Motivation, sich auch in Zukunft für ihr Dorf einzusetzen, weil sie eine Erfolgserwartung entwickelt haben. Die Themenbereiche, in denen sich die Menschen engagieren, sind vielfältig: Zum Teil geht es um das Instandhalten oder Neuschaffen öffentlicher Plätze und Treffpunkte (Neu- 
vrees), zum Teil werden Feste organisiert (Bodensee), zum Teil werden auch größere Projekte neu erdacht wie etwa das Angebot eines Mittagstisches (Hemeln). Zudem wird ein Engagement auch dadurch motiviert, dass seitens einer übergeordneten politischen Gemeinde wenig ,passiert . Hier steht dann zum Teil eine Frustration über die vorhandenen Bedingungen im Mittelpunkt. Gekoppelt mit einer hohen (kollektiven) Selbstwirksamkeitserwartung kommt es dann zu einem Engagement für bessere Bedingungen.

\subsection{Reaktionen auf den demographischen Wandel und Meinung zu neuen Dorfentwicklungsprozessen}

Ein Bewusstsein für den demographischen Wandel ist eher im Süden Niedersachsens vorhanden als im Nordwesten, da Leerstand und damit verbundene Probleme hier schon gegenwärtiger sind als im Nordwesten. Aber auch im Süden des Landes gibt es noch Stimmen, die meinen, der demographische Wandel finde woanders, aber nicht bei ihnen statt. Reaktionen auf den demographischen Wandel finden sich dementsprechend auch vermehrt im Landkreis Göttingen: Hier gibt es in den Dörfern bereits Leerstandskataster und andere kreative Lösungen - oft initiiert von Bürgern -, um die Innenentwicklung zu fördern. Im Nordwesten hingegen wird kaum von nicht vermittelbarem Leerstand, sondern eher von Bedarf nach Bauplätzen für Neubauten berichtet, gerade auch, weil hier die jungen Erwachsenen häufiger im Dorf bleiben oder dorthin zurückkommen.

Die Idee der Dorfmoderation aus Göttingen, wie sie oben geschildert wurde, ist zum Zeitpunkt der Interviews noch nicht sehr bekannt. Selbst die Bewohner der Dörfer im Landkreis Göttingen können nur zum Teil etwas mit dem Begriff anfangen. In dem Dorf, in dem es eine Dorfmoderatorin gibt, ist die Beurteilung ausnahmslos positiv und auch das Verhältnis zwischen Bürgermeister und Dorfmoderatorin wird als gut und konkurrenzlos beschrieben. Im zweiten untersuchten Dorf des Landkreises Göttingen ist das Angebot zur Weiterqualifizierung relativ unbekannt, wird aber als Idee positiv aufgenommen, weil der Bedarf einer neutralen Unterstützung für die Dörfer sehr wohl gesehen wird. In den beiden Dörfern im Nordwesten Niedersachsens blickt man eher skeptisch auf eine solche Idee und meint, man habe „das Dorfleben selbst im Griff“"

Keine einheitliche Antwort gab es auf die Frage, ob Dorfmoderatoren aus dem eigenen Dorf oder von außerhalb kommen sollten. Dorfbewohner, die bereits Erfahrung mit einer Dorfmoderation aus ihrer Nachbarschaft gemacht haben, befürworteten eher dieses Modell. Insbesondere die ständige Ansprechbarkeit direkt ,über den Gartenzaun“ und die Ortskenntnisse waren hier überzeugend.

Als Idee zur Legitimierung eines zusätzlichen Kümmerers oder Moderators wurde genannt, man könnte so jemanden eventuell gleichstellen mit dem Amt des Ortsheimat- pflegers oder ein Dorfmoderator sollte mit in die Sprechstunde des Bürgermeisters kommen, um dort vor Ort mit Bürgern sprechen und direkt von deren Wünschen und Problemen erfahren zu können. Insgesamt zeigen die bisher gemachten Erfahrungen mit Dorfmoderatoren aus dem Landkreis Göttingen, dass eine Zusammenarbeit mit dem Ortsrat gut möglich ist, wenn Offenheit und ein guter Wille da sind.

\subsection{Lebensgefühl im Dorf auf den Punkt gebracht}

Gefragt nach dem größten Vorteil des Dorflebens nennen die Menschen in den Dörfern übereinstimmend zwei große Bereiche: Zum einen werden die Natur, die Naherholung, der Garten und der viele Platz hervorgehoben. Zum anderen werden die vertrauten Menschen, die Gemeinschaft, die wie eine große Familie sei, betont. Diese Gemeinschaft ist eine Ressource, die gekoppelt mit der hohen Selbstwirksamkeitserfahrung wiederum ein bürgerschaftliches Engagement für das Dorf unterstützen kann, was dann besonders wirksam wird, wenn es durch moderierende Ansätze unterstützt wird.

\section{Diskussion und Schlussfolgerungen}

Die Forschungsergebnisse zeigen, dass die Lebensqualität in Dörfern insbesondere an folgenden drei Dimensionen festgemacht werden kann:

- an der Qualität der natürlichen Umgebung (Naturnähe),

- an den ausgeprägten sozialen Beziehungen (Dorfgemeinschaft) und

- an den vielfältigen Mitgestaltungsmöglichkeiten (Selbstwirksamkeitserfahrung).

Vieles ist nicht neu. So hat auch Krambach (2013) diese Qualitäten als die Vorzüge des Dorfes gegenüber der Stadt beschrieben, und Ritzinger (2011) hat darauf hingewiesen, dass neue Beteiligungsstrukturen ein Schlüssel für eine erfolgreiche Dorfentwicklung sind. Unsere Forschungsergebnisse bestätigen nicht nur diese Aussagen, sondern heben die Bedeutung sozialer Themen der Dorfentwicklung in Bezug auf die Bewahrung und Schaffung einer guten Lebensqualität hervor. Es wird weiterhin deutlich, dass die Dorfbewohner in der aktiven Mitgestaltung eine gute Möglichkeit sehen, zur Bewahrung und Schaffung einer guten Lebensqualität beizutragen. Diese Möglichkeiten tragen einerseits über eine erhöhte Selbstwirksamkeitserfahrung zur eigenen Lebenszufriedenheit bei, andererseits stellen sie die Basis für die gemeinschaftliche Gestaltung und Schaffung einer guten Lebensqualität im Dorf dar. Damit lassen sich die in Kapitel 2.3 beschriebenen Aussagen und Befunde von Herriger (2006), Eigner-Thiel (2005) und Gensicke und Geiss (2010) zu Motiven und Konsequenzen von bürgerschaft- 
lichem Engagement bestätigen. Die quantitativen Befunde von Freitag, Rößler und Kunz (2009) zur Lebensqualität im Dorf werden durch unsere Studie ebenfalls bestätigt und darüber hinaus wird deutlich, warum der Zusammenhalt im Dorf so attraktiv ist: Dass man nämlich gemeinschaftlich auch etwas bewirken kann, um die eigenen Lebensbedingungen zu verbessern. Dies wiederum kann, wie eingangs dargestellt, die psychische Gesundheit der Individuen im Dorf langfristig positiv beeinflussen (vgl. Bengel/ Strittmatter/Willmann 2001).

Was bedeutet das nun für die Dorfentwicklung? Der hier verwendete Begriff „Dorfentwicklung“ bezieht neben Dorferneuerungsverfahren auch neue Dorfentwicklungsprozesse mit ein. In Anknüpfung an Magel (2011: 123) geht es in Dorferneuerungsverfahren in Zukunft insbesondere darum, „Bürger nicht nur zu beteiligen, sondern ihnen konkrete Verantwortung zu übergeben“. Unsere Forschungsergebnisse zeigen, dass Dorfbewohner heute in vielen Handlungsfeldern, auch unabhängig von Dorferneuerungsverfahren, eine Mitverantwortung für die Bewahrung und Schaffung von Lebensqualität im Dorf übernehmen. Das zivilgesellschaftliche Potenzial des Dorfes verdient daher eine stärkere Beachtung in der Dorferneuerung. In diesem Zusammenhang weist Ritzinger (2011: 88) zu Recht darauf hin, dass für eine erfolgreiche Weiterentwicklung der Dorferneuerung vor allem neue methodische Vorgehensweisen wichtig sind, die die lokalen Akteure als eine Verantwortungsgemeinschaft in den Dorfentwicklungsprozess einbezieht. Damit das gelingen kann, sollte gleich zu Beginn jedes Dorferneuerungsverfahrens in die Menschen und erst dann in Gebäude, Wege und Plätze investiert werden (Karweik 2009: 33). Es sind also weniger die neuen Themen, die eine Herausforderung für die Dorferneuerung darstellen, sondern vielmehr die Methoden der Umsetzung. Diese fehlen bisher oder werden noch zu wenig genutzt.

Eine methodische Weiterentwicklung der Dorferneuerung allein reicht aber nicht aus, um langfristige Dorfentwicklungsprozesse im Sinne des Aufbaus einer Verantwortungsgemeinschaft zu etablieren: Die von uns analysierten Beziehungen zwischen der Lebensqualität und Dorfentwicklungsprozessen unterstreichen die Bedeutung von organisatorisch und finanziell unterstützenden Strukturen für die Verstetigung von Dorfentwicklungsprozessen, die auch Ritzinger (2011: 88) fordert. Die vertraute Dorfgemeinschaft und eine über Jahre entwickelte hohe (kollektive) Selbstwirksamkeitsüberzeugung werden in diesem Kontext als wichtige endogene Potenziale bewertet, die ihre Wirkung aber nur dann voll entfalten können, wenn der Entwicklungsprozess zielgerichtet organisiert wird. Die Entwicklung dorfbezogener Governancen (neue Dorfvereine), die in Kapitel 2.2 (vgl. Born 2009) beschrieben wurden, stellen eine Möglichkeit dar, bürgerschaftliches Engagement für das Dorf zielorientiert zu bündeln. Wenn dies geschieht oder damit dies leichter geschieht, kann eine Unterstützung durch eine neutrale Instanz (z. B. einen Moderator, einen Kümmerer, eine Dorfgesprächsrunde) sinnvoll sein, die wiederum möglichst finanziell und ideell auch durch übergeordnete Strukturen wie die Gemeinde oder den Landkreis mitgetragen wird.

An diese Überlegung knüpft das Qualifizierungsangebot zum Dorfmoderator an, welches aktuell im Landkreis Göttingen erprobt und weiterentwickelt wird (vgl. Kapitel 2.2). Die Ergebnisse des Forschungsvorhabens „Lebensqualität im Dorf" unterstreichen, dass die Erhaltung der Lebensqualität im eigenen Dorf ein starkes und leitendes Motiv für das Engagement der Dorfbewohner ist. Die zunehmenden Probleme in vielen Dörfern erhöhen den Handlungsdruck und damit verbunden zunehmend auch die Bereitschaft bei allen Beteiligten, neue Wege der Dorfentwicklung zu gehen. Die Einbindung der Dorfbevölkerung ist zwar eine Grundvoraussetzung für die Zukunftsentwicklung der Dörfer, aber kein Allheilmittel. Sie entbindet den Staat nicht von seinen Aufgaben: Die Dorferneuerung bleibt immer auch eine öffentliche Aufgabe, für die ausreichende Mittel zur Verfügung gestellt werden müssen. In der Bündelung der Kräfte von Zivilgesellschaft und Staat liegt jedoch die Chance, das Dorf als traditionsreiche und wertgeschätzte Siedlungseinheit zu erhalten. Die Dorfentwicklung zu Beginn des 21. Jahrhunderts sollte sich daher im besten Sinne als eine experimentierfreudige Anpassungsplanung verstehen und entsprechende inhaltliche Akzente setzen, sich aber auch methodisch weiterentwickeln.

Bezüglich der methodischen Vorgehensweise soll hier selbstkritisch die Auswahl der Interviewteilnehmer reflektiert werden. Diese wurden in den entfernteren Dörfern vom jeweiligen Ortsvorsteher ausgesucht. Letztlich ist diese Auswahl subjektiv und eventuell verfolgten die Repräsentanten eines Dorfes auch die Absicht, ihr Dorf möglichst positiv darzustellen, was die Auswahl ihrer vorgeschlagenen Interviewpartner mit beeinflusst haben könnte. Denn man gewinnt den Eindruck, dass die Gruppe der Interviewten trotz äußerlich heterogener Merkmale (wie z. B. Alter) relativ homogen und im Sinne eines, vorbildlichen Dorfes ' antworteten. Hier sollte in Folgestudien im Sinne der Grounded Theory versucht werden, sich nach den ersten Interviews selbst weitere Interviewpartner im Dorf zu suchen. Dies kann organisatorisch etwas schwieriger sein; trotzdem sollte dieser Aspekt bei der Interpretation der Ergebnisse berücksichtigt werden. Abgesehen davon hat sich das qualitative Vorgehen sehr bewährt, weil Zusammenhänge und Begründungen deutlich geworden sind, die in einem quantitativ auszuwertenden Fragebogen nicht zu Tage getreten wären.

Die Verknüpfung des psychologischen Konzepts der Lebensqualität mit raumwissenschaftlichen Betrachtungsweisen stellte eine besondere methodische Herausforderung 
dar. Im Ergebnis wird aber deutlich, dass die raumwissenschaftliche Forschung wichtige Impulse aus der Psychologie aufnehmen kann. Die Übertragung der Ergebnisse der Untersuchung in Handlungsstrategien der Dorfentwicklung wird diskutiert, aber es bedarf weiterer interdisziplinärer Forschungsarbeit, um neue umsetzungsorientierte Methoden zu entwickeln und diese dann in der Praxis zu erproben.

\section{Literatur}

Bandura, A. (1991): Social Cognitive Theory of Self-Regulation. In: Organizational Behavior and Human Decision Processes 50, 2, 248-287.

Becker, U. (2012): Lebensqualität für zukunftsfähige Städte und Dörfer. In: Land Brandenburg (Hrsg.): Workshop Lebensqualität für zukunftsfähige Städte und Dörfer. Potsdam, 10-12. http://www. mlul.brandenburg.de/cms/media.php/lbm1.a.3310.de/doku_ws_ lebensqualitaet.pdf (08.06.2016).

Bengel, J.; Strittmatter, R.; Willmann, H. (2001): Was erhält Menschen gesund? Anotonovskys Modell der Salutogenese - Diskussionsstand und Stellenwert. Köln. = Forschung und Praxis der Gesundheitsförderung 6.

Berlin-Institut für Bevölkerung und Entwicklung (2015): Von Hürden und Helden. Wie sich das Leben auf dem Land neu erfinden lässt. Berlin.

BMFSFJ - Bundesministerium für Familie, Senioren, Frauen und Jugend (2013) (Hrsg.): Motive bürgerschaftlichen Engagements. Kernergebnisse einer bevölkerungsrepräsentativen Befragung durch das Institut für Demoskopie Allensbach im August 2013. Berlin.

Born, K. M. (2009): Anpassung und Governance im Dorf. In: Ländlicher Raum 60, 3, 58-61.

Born, K. M. (2011): Ländliche Räume in Deutschland. Differenzierungen, Entwicklungspfade und -brüche. In: Geographische Rundschau 63, 2, 4-11.

Ebbinghaus, B.; Noll, H-H.; Bahle, T.; Wendt, C.; Scheuer, A. (2006): VFA-Report Lebensqualität in Deutschland 2006. Mannheim. http://www.mzes.uni-mannheim.de/publications/papers/VFAReport_2006II.pdf (08.06.2015).

Eigner-Thiel, S. (2005): Kollektives Engagement für die Nutzung erneuerbarer Energieträger - Motive, Mobilisierung und Auswirkungen am Beispiel des Aktionsforschungsprojekts „Das Bioenergiedorf". Hamburg.

Eigner-Thiel, S.; Meyer, R. (2015): Alle kommen zu Wort Moderation in der Dorfentwicklung. Dokumentation der Tagung vom 19.-21.4.2015 in Göttingen. Göttingen. http:// www.landkreisgoettingen.de/pics/medien/1_1457614612/ Dokumentation_DM-Tagung_2015.pdf (08.06.2016).

Freitag, T.; Rößler, C.; Kunz, A. (2009): Daseinsvorsorge aus Bürgersicht. Bewertung der Lebensverhältnisse und Daseinsvorsorge vor dem Hintergrund der infrastrukturellen Ausstattung sächsischer Dörfer. Dresden. $=$ Schriftenreihe des Landesamtes für Umwelt, Landwirtschaft und Geologie 31.

Gensicke, T.; Geiss, S. (2010): Hauptbericht des Freiwilligensurveys 2009. Ergebnisse der repräsentativen Trenderhebung zu Ehrenamt, Freiwilligenarbeit und Bürgerschaftlichem Engagement. München.

Glaser, B.G.; Strauss, A. L. (1998): Grounded Theory. Strategien qualitativer Forschung. Bern.

Habich, R.; Spellerberg, A. (2013): Regionale Disparitäten. In: Statistisches Bundesamt; Wissenschaftszentrum Berlin für Sozialforschung (Hrsg.): Datenreport 2013 - Ein Sozialbericht für die Bundesrepublik Deutschland. Berlin, 310-322.
Hauptmeyer, C.-H. (1983): Zukunftsperspektiven des Dorfes - historische Aspekte. In: Hauptmeyer, C.-H.; Henckel, H.; Ilien, A.; Reinecke, K.; Wöbse, H. H. (Hrsg.): Annäherungen an das Dorf. Geschichte, Veränderung und Zukunft. Hannover, 200-216.

Henkel, G. (1995): Der Ländliche Raum: Gegenwart und Wandlungsprozesse seit dem 19. Jahrhundert in Deutschland. Stuttgart.

Henkel, G. (2012): Das Dorf. Landleben in Deutschland - gestern und heute. Stuttgart.

Herriger, N. (2006): Empowerment in der Sozialen Arbeit: Eine Einführung. Stuttgart.

Hunecke, M. (2013): Psychische Ressourcen zur Förderung nachhaltiger Lebensstile. Memorandum des Denkwerks Zukunft - Stiftung kulturelle Erneuerung. Bonn.

Karweik, K. (2009): VIP für VIPs in der Dorferneuerung - vom Modellprojekt zum Erfolgsmodell. In: Ländlicher Raum 60, 3, 2933.

Krambach, K. (2013): Dorfbewegung - warum und wie? Berlin. http:// www.rosalux.de/fileadmin/rls_uploads/pdfs/rls_papers/Papers_ Dorfbewegung.pdf (08.06.2016).

Küpper, P.; Scheibe, C. (2015): Steuern oder fördern? Die Sicherung der Nahversorgung in den ländlichen Räumen Deutschlands und Südtirols im Vergleich. In: Raumforschung und Raumordnung 73, 1, 45-58.

Kunz, A.; Schumacher, U. (2010): Dorf-GERECHT ... Lebensqualität neu organisieren - Beispiele aus Sachsen. In: DLKG - Deutsche Landeskulturgesellschaft (Hrsg.): Dörfer ohne Menschen!? Zwischen Abriss, Umnutzung und Vitalisierung und Mehr Dorf für weniger Bürger - Chancen und Perspektiven für ländliche Siedlungen. Müncheberg, 53-68. = Schriftenreihe Deutsche Landeskulturgesellschaft DLKG 7.

Legewie, H.; Janßen, M. (1997): Bürgerinitiativen fördern Gesundheit in der Stadt. In: Klotter, C. (Hrsg.): Prävention im Gesundheitswesen. Göttingen, 326-356.

Magel, H. (2000): Zusammenfassung und Ausblick. In: Magel, H. (Hrsg.): Dorferneuerung vor neuen Herausforderungen. München, 133-138. = Materialiensammlung des Lehrstuhls für Bodenordnung und Landentwicklung der TU München 24.

Magel, H. (2011): Schrumpfung bleibt eine große Herausforderung. Zusammenfassung. In: Magel, H. (Hrsg.): Schrumpfung als Herausforderung für ländliche Räume. München, 121-124. = Materialiensammlung des Lehrstuhls für Bodenordnung und Landentwicklung der TU München 43.

Magel, H.; Bock, H. (2007): Eine einmalige Erfolgsgeschichte fortsetzen - Zum Selbstverständnis der Dorferneuerung in Bayern. In: Politische Studien 58, 2, 138-149.

Mayring, P. (2002): Einführung in die qualitative Sozialforschung. Weinheim.

Niedersächsischer Heimatbund; Niedersächsische Akademie ländlicher Raum (2014): Zur Zukunft der Dörfer in Niedersachsen. Positionspapier des Niedersächsischen Heimatbundes e.V. und der Niedersächsischen Akademie ländlicher Raum e.V. zu Leitlinien künftiger Dorfentwicklung. Hannover.

Noll, H.-H. (2000): Konzepte der Wohlfahrtsentwicklung: Lebensqualität und "neue" Wohlfahrtskonzepte. Berlin. = WZB Discussion Paper P 00-505.

Ritzinger, A. (2011): Forschungsprojekt „Dorferneuerung 2020“: Neue Schwerpunkte in strukturschwachen, schrumpfenden Dörfern. In: Magel, H. (Hrsg.): Schrumpfung als Herausforderung für ländliche Räume. München, 81-89. = Materialiensammlung des Lehrstuhls für Bodenordnung und Landentwicklung der TU München 43.

Schmitz, G. S.; Schwarzer, R. (2002): Individuelle und kollektive Selbstwirksamkeitserwartung von Lehrern. In: Jerusalem, M.; Hopf, D. (Hrsg.): Selbstwirksamkeit und Motivationsprozesse in Bildungsinstitutionen. Weinheim, 192-214. = Zeitschrift für Pädagogik, Beiheft 44.

Schwarzer, R. (2004): Psychologie des Gesundheitsverhaltens. Einführung in die Gesundheitspsychologie. Göttingen. 
Spellerberg, A.; Huschka, D.; Habich, R. (2006): Quality of life in rural areas. Processes of divergence and convergence. http://www.soc. duke.edu/resources/sinet/papers06/Spellerberg.pdf (08.06.2016).

Steinführer, A. (2015): Bürger in der Verantwortung. Veränderte Akteursrollen in der Bereitstellung ländlicher Daseinsvorsorge. In: Raumforschung und Raumordnung 73, 1, 5-16.

Strauss, A. L.; Corbin, J. M. (1990): Basics of qualitative research. Grounded Theory procedures and techniques. Newbury Park.
Troßbach, W.; Zimmermann, C. (2006): Die Geschichte des Dorfes. Von den Anfängen im Frankenreich bis zur bundesdeutschen Gegenwart. Stuttgart.

Wilde, M. (2014): „Ach, da fahr ich ganz spontan.“ Mobilität im Alltag älterer Menschen auf dem Land. In: Raumforschung und Raumordnung 72, 5, 371-384.

Witzel, A. (2000): Das problemzentrierte Interview. In: Forum Qualitative Sozialforschung, 1, 1, Artikel 22. http://nbn-resolving.de/ urn:nbn:de:0114-fqs0001228 (08.06.2016). 